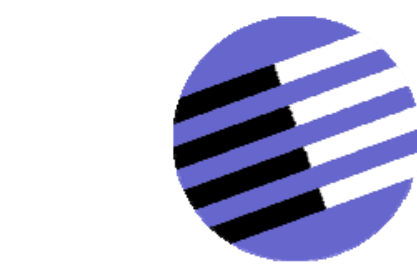

GOVERNANCE AND THE EFFICIENCY

OF ECONOMIC SYSTEMS

GESY

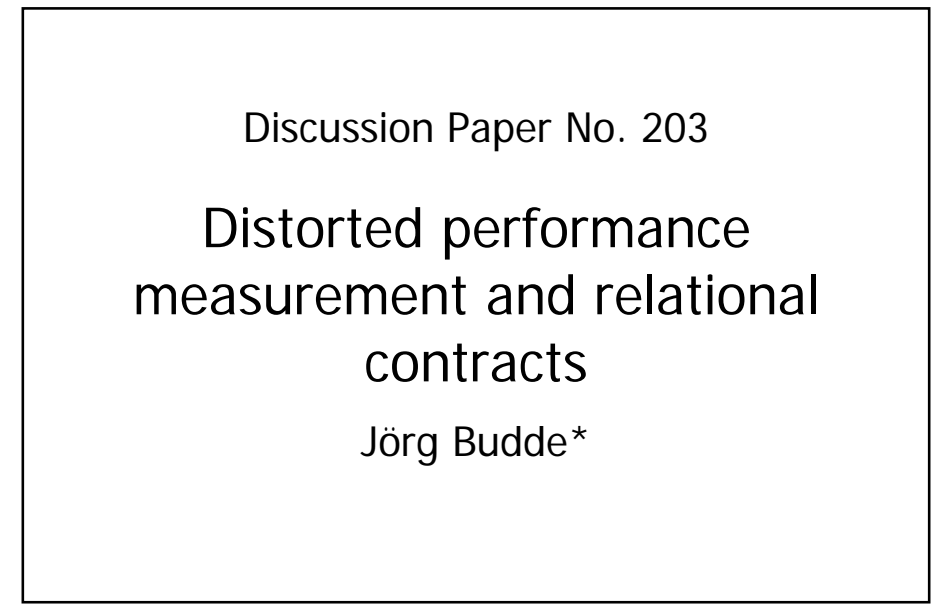

November 2006

*Jörg Budde, Jörg Budde, Department of Economics, University of Bonn, Adenauerallee 24-42,

D-53113 Bonn; phone: +49-228-739247,

Joerg.Budde@uni-bonn.de

Financial support from the Deutsche Forschungsgemeinschaft through SFB/TR 15 is gratefully acknowledged.

Sonderforschungsbereich/Transregio $15 \cdot$ www.gesy.uni-mannheim.de

Universität Mannheim · Freie Universität Berlin · Humboldt-Universität zu Berlin · Ludwig-Maximilians-Universität München Rheinische Friedrich-Wilhelms-Universität Bonn · Zentrum für Europäische Wirtschaftsforschung Mannheim 


\title{
Distorted performance measurement and relational
} contracts

\author{
Jörg Budde ${ }^{\S}$
}

November 30, 2006

\footnotetext{
¥The authors likes to thank Christian Riegler and participants of the GEABA symposium 2006 in Bielefeld for helpful comments. Financial support by the Deutsche Forschungsgemeinschaft through SFB/TR 15 is gratefully acknowledged.

$\S$ Address of Correspondence: Jörg Budde, Department of Economics, University of Bonn, Adenauerallee 24-42, D-53113 Bonn; phone: +49-228-739247; email: Joerg.Budde@uni-bonn.de
} 


\title{
Distorted performance measurement and relational contracts
}

\begin{abstract}
This paper analyzes the use of alternative performance measures in an agency model in which contracting incorporates both formal and informal agreements. It is shown that under a proper use of verifiable and unverifiable performance measures, the two types of contracts are complements, regardless of the principal's fallback position. The analysis therefore contrasts earlier results of the literature, and provides a rationale for the application of subjective performance information, as it is frequently incorporated in strategic performance measurement systems.
\end{abstract}




\section{Introduction}

In the last decade, strategic performance measurement has become an increasingly popular device for both decision facilitating and incentive purposes. Triggered by the insight that in a changing environment, traditional financial measures may not suffice to properly represent a firm's actual condition, several concepts have been proposed which try to capture the long-term effects of managerial activities. In most of these concepts, the use of non-financial measures is suggested to cover these effects. ${ }^{1}$

At first, the main objective of these systems was to identify, communicate and implement the firm's goals within the organization. Corporate practice, however, has meanwhile lead to the understanding that the strategy cannot be enforced without tying employees' compensation to respective measures (e.g., Kaplan and Norton 2001, 151). A potential deficiency in this respect is that non-financial information need not be verifiable to a third party, and therefore at the outset cannot be assumed to be applicable to formal contracting. Alternatively, it can be used as a basis of subjective rewards, which may be combined with a formal contract based on a distorted financial performance index.

In this paper, I revisit the question of how these two types of contracts are optimally combined. To this purpose, I adapt a model of relational contracts proposed by Baker et al. (1994) to a multi-task agency setting. I extend their work by considering a different arrangement in which the relational contract is used not in addition to the formal contract, but in exchange to (part of) it. This modification considerably changes the results. Although it proves true that subjective rewards

\footnotetext{
${ }^{1}$ For example, the balanced scorecard as the most popular example of a strategic performance measurement system complements its financial measures by three further perspectives (customer, internal and learning and growth) which may incorporate measures such as customer satisfaction or employee loyalty. For details, see Kaplan and Norton $(1992,1996)$.
} 
will not always be credible, the more elaborate contract is credible for a much wider range. As a consequence, the contrariness of the results of Baker et al. is removed since under the more subtle arrangement, formal and relational contracts work as complements, regardless of the principal's fallback position.

The remainder of this paper is structured as follows: Section 2 describes the stage game of a one-period agency framework and analyzes the optimal formal contract. Section 3 provides a multi-period extension of the initial agency model and studies the use of subjective rewards. Section 4 draws conclusion concerning the requirements for strategic performance measures. All proofs are in the appendix.

\section{One-period framework}

In this section I describe a one-period agency model with distorted financial performance measurement which will serve as the stage game in the multi-period environment of the next section. Contrary to Baker et al. (1994), I assume that performance measure distortion does not arise from asymmetric information, but from the multiplicity of tasks the agent has to perform. Formally, both scenarios are much alike (see Holmström and Milgrom 1991). The multi-task agency, however, allows to refer more clearly to an index of performance measure congruity proposed by Baker (2000, 2002). By a slight modification of this index, the impact of congruity on the interplay of formal and relational contracts will become particularly apparent when the multi-period model is studied.

So as a starting point, consider a situation in which a principal hires an agent to work one-time on his behalf. The agent's activity $\mathbf{a} \in \mathbb{R}^{n}$ has multiple aspects and cannot be legally enforced. The principal seeks to maximize his value $V$ from the agent's action, net of wage payments. $V(\mathbf{a})=\mathbf{d}^{\prime} \mathbf{a}$ is assumed to be a linear function 
of the agent's action $\mathbf{a}$, in which $\mathbf{d}=\left(d_{i}, \ldots, d_{n}\right)^{\prime}$ is the vector of the marginal products of the different activities. $V$ may be interpreted as the agent's contribution to firm value. In order to analyze the problem of performance measurement distortion in formal contracts, I assume that $V$ can be observed by both parties, but that it is not verifiable to an outside party. Thus, no formal contract may be based on $V$.

Since neither a nor the principal's objective $V$ can be contracted on, the principal has to rely on a performance measure $P(\mathbf{a})=\mathbf{y}^{\prime} \mathbf{a}+\epsilon=\sum_{i=1}^{n} y_{i} a_{i}+\epsilon$ in order to motivate the agent for the activity. $y_{i} \in \mathbb{R}$ denotes the performance measure's sensitivity $^{2}$ with respect to action $a_{i}$, and $\epsilon \sim N\left(0, \sigma^{2}\right)$ is a normal error term reflecting the uncertainty related to measure $P$. With regard to the above mentioned issue of strategic performance measurement, $P$ represents the (aggregate of) financial performance measure(s), which does not capture all relevant aspects of the firm's performance. Thus $\mathbf{y} \neq \mathbf{d}$.

Based on $P$, the principal offers a linear incentive contract $S=s_{b}+s_{p} P$ in which $s_{b}$ is a base salary and $s_{p}$ is the share parameter defining the performance related payment.

By choosing a, the agent incurs a private cost $C(\mathbf{a})$. Similar to most treatises of the linear agency framework, I assume that $C$ is of the form $C(\mathbf{a})=\frac{1}{2} \mathbf{a}^{\prime} \mathbf{a}$ (Cf., for example, Itoh 1991, Feltham and Xie 1994, or Baker 2000). ${ }^{3}$ In order to abstract from risk sharing issues, both principal and agent are assumed to be risk neutral. ${ }^{4}$ The agent's utility from his compensation $S$ and his action choice a is given by

\footnotetext{
${ }^{2}$ See Banker and Datar (1989) for a definition.

${ }^{3}$ In order to account for task complementarities or substitutabilities, one could also consider $C$ to be a quadratic form $\mathbf{a}^{\prime} \mathbf{C a}$, which would proxy general cost functions as considered by Holmström and Milgrom (1991). The resulting effects, however, could likewise be captured by a redefinition of tasks, resulting in modified marginal products $\mathbf{d}$.

${ }^{4}$ One could ask why the principal does not sell the firm to the manager. However, $V$ not necessarily needs to be the value of the firm as a whole. It may also represent the value added by the manager, which cannot be sold because it is not separable from the remaining assets of the firm.
} 
$U^{A}(S, \mathbf{a})=S-C(\mathbf{a})$. His outside options are incorporated by a reservation utility $U^{R} \geq 0$.

The principal's contracting problem in this model is a special case of that analyzed by Feltham and Xie (1994), who allow for a risk-averse agent and multiple performance measures. Consequently, the optimal contract can directly be derived from their analysis. By choosing $s_{p}$, the principal maximizes the expected total surplus $\Pi=V(\mathbf{a})-C(\mathbf{a})$, subject to the the incentive compatibility constraint $\mathbf{a}=s_{p} \mathbf{y}$. The base salary $s_{b}$ is chosen to ensure that the agent's reservation level of utility is obtained. The optimal share parameter is ${ }^{5}$

$$
s_{p}^{0}=\frac{\mathbf{d}^{\prime} \mathbf{y}}{\mathbf{y}^{\prime} \mathbf{y}} .
$$

from which the agency's net total surplus becomes

$$
\Pi^{0}=\Pi\left(s_{p}^{0}\right)=\frac{1}{2} \frac{\left(\mathbf{d}^{\prime} \mathbf{y}\right)^{2}}{\mathbf{y}^{\prime} \mathbf{y}} .
$$

Total profit equals $\left(\mathbf{y}^{0}\right)^{\prime} \mathbf{y}^{0} / 2$, where $\mathbf{y}^{0}=s_{p}^{0} \mathbf{y}$ is the sensitivity of the scaled performance measure $P^{0}=s_{p}^{0} P$. Among all implementable actions $s_{p} \mathbf{y}, \mathbf{a}^{0}=\mathbf{y}^{0}$ describes the one which is "closest" to the first-best action $\mathbf{a}^{F B}=\mathbf{d}$. The alignment of $\mathbf{y}$ and $\mathbf{d}$ is referred to as a the congruity of the performance measure $P$, for which a number of metrics has been suggested (Feltham and Xie 1994, Feltham and Wu 2000, Datar et al. 2001). By inspection of (1), it is most promising to refer to Baker (2000, 2002), who proposes the cosine of the angle between the vectors $\mathbf{d}$ and $\mathbf{y}$ as a metric of congruity. Since the cosine can be written as

$$
\cos (\mathbf{d}, \mathbf{y})=\frac{\mathbf{d}^{\prime} \mathbf{y}}{\sqrt{\mathbf{d}^{\prime} \mathbf{d}} \sqrt{\mathbf{y}^{\prime} \mathbf{y}}},
$$

\footnotetext{
${ }^{5}$ See Feltham and Xie 1994, p. 433.
} 
it naturally relates the total surplus (2) to the first-best total surplus, which is given by $\Pi^{F B}=\frac{1}{2} \mathbf{d}^{\prime} \mathbf{d}$. The relation is formulated in the following lemma:

Lemma 1 The total surplus in a risk-neutral linear agency model with quadratic effort cost is given by

$$
\Pi^{0}=(\cos (\mathbf{d}, \mathbf{y}))^{2} \Pi^{F B}
$$

Proof The relation directly follows from a computing $\Pi^{0} / \Pi^{F B}$

For notational convenience, I will denote $\phi(\mathbf{d}, \mathbf{y})=(\cos (\mathbf{d}, \mathbf{y}))^{2}$ as the congruity of performance measure $P$ with respect to the firm's objective $V$. By squaring, it scales the cosine measure to the unit interval, and the second-best total profit $\Pi\left(\mathbf{s}^{0}\right)=\phi \Pi^{F B}$ becomes a linear function of congruity. This will greatly facilitate the notation of the multi-period model in section 3 .

\section{Relational contracts}

\subsection{Basic idea}

As long as the performance measure applicable in a formal contract is not perfectly congruent with the firm's objectives, the question arises whether the non-verifiable information can be used to improve the contract. One instrument to do this are relational contracts. Since these "informal agreements and unwritten codes of conduct" (Baker et al. 2002) are not meant to be enforced by law, they can be based on outcomes that are observed ex post by the contracting parties alone. Obviously, relational contracts are only of substance if it is in both parties' best interest to keep the agreement. In the one-shot relationship analyzed in the preceding section, this will never be the case: the principal will always gain from refusing a voluntary payment. Consequently, relational contracts are usually analyzed in a multi-period 
framework in which the parties repeatedly agree upon a contract. I take this approach in order to analyze how subjective rewards can be used to improve a formal contract.

In doing so, I adapt a model of Baker et al. (1994) who analyze how formal and relational contracts can optimally be combined in an infinitely repeated agency relationship. They assume that the subjective assessment of firm value is complemented by a distorted verifiable performance measure. In their model, distortion stems from the assumption that the sensitivity of the performance measure is privately observed by the agent after contracting. Two payments are determined ex ante: a piece rate from the formal contract which is based on the verifiable measure, and a bonus from the relational contract which is based on the non-verifiable subjective evaluation.

Analyzing the combination of these two contracts, Baker et al. show that the existence of a sufficiently congruent verifiable performance measure may rule out any relational agreement. The reason of this negative result is convincing: since the bonus is voluntarily payed, it is subject to a credibility constraint. It must be in the principal's best interest to pay. Under the assumption that both parties apply Grim-trigger strategies in the repeated game ${ }^{6}$ this is the case if the amount the principal could save by refusing the bonus does not exceed his future benefits from an ongoing relational contract. This benefit depends on the principal's profit after a potential defection. Baker et al. assume that the agent still accepts the formal contract. ${ }^{7}$ Consequently, the principal's fallback position critically depends on the congruity of the verifiable measure: the more congruent this measure is, the less the

\footnotetext{
${ }^{6}$ In broad terms, this strategies can be described as follows: both parties employ cooperative strategies (work hard and pay the bonus) as long as no party has defected, and continue by noncooperation as soon as one party breaks the agreement. Although this is not a unique equilibrium of the dynamic game, the assumption is without loss of generality with respect to the obtainable payoffs (see Abreu 1988).

${ }^{7}$ This assumption is critical for their negative results. Thus, to obtain comparability, I will stick to this assumption in the following analysis.
} 
principal benefits from maintaining the relational agreement.

In my view, the analysis of Baker et al. stresses one side of the coin because congruity may also reduce the payable bonus. Incentives already provided by a formal contract need not be sustained by the relational agreement. Thus, if the verifiable performance measure is almost perfectly congruent, only little fine-tuning should be due to subjective rewards. Of course, a proper coordination of the two contracts is essential for that purpose. In this respect, the agreement considered by Baker et al. is rather coarse: the bonus is based exclusively on the realization of firm value although the verifiable measure $P$ could also be taken into account. In the following, I will analyze such more elaborate contracts.

The most apparent way to incorporate $P$ into the relational contract would be to offer a bonus for a target of an aggregate measure derived from $V$ and $P$. This approach is simlar to that taken in Budde (2006), where a system $\mathbf{P}=\left(P_{1}, \ldots, P_{m}\right)$ of partly unverifiable measures is studied. It emerges that if $\mathbf{P}$ is capable of mimicing the principal's objective $V$, the optimal relational contract is build on an aggregate measure $V-P^{0}$, while the second-best contract $s^{0}$ is offered as a formal contract. That way, subjective rewards exactly provide incentives for the effort gap $\mathbf{d}-\mathbf{y}^{0}$ not provided by the formal contract, and the bonus is kept to a minimum. The result, however, builds on the assumption that $\mathbf{P}$ can subjectively be assessed without noise. Only then the agent can be sure to receive the bonus if he acts according to the agreement. Under noisy performance measurement, the bonus is uncertain, and its required amount becomes a matter of congruity and precision. Thus, although the same logic should apply here, the results cannot directly be transferred to the present setting.

The same coordination, however, can also be achieved by a different agreement which does not require the precise assessment of the agent's impact on measure $P$. 
The key idea is to combine subjective rewards not with the realized amount of the agent's piece rate, but with its expected amount. This is possible if the verifiable measure has not been realized when the bonus is paid. Thus in the analysis, I assume the following timeline of events in each period $t$ of the dynamic game:

[Figure 1 about here.]

This timeline is not unrealistic. Financial measures, such as accounting income, usually suffer from late issue because accounts are settled not until the end of the accounting period. Subjective evaluation, in contrast, is not subject to such terms and may be available as soon as the agent has completed his tasks.

Given this progression, the effects of a bonus based on $V-P^{0}$ can be reproduced by the following agreement:

1. At date t.0, the two parties sign the formal contract $\left(s_{b}, s_{p}^{0}\right)$. This contract is augmented by a clause that up to date $t .2$, the principal may fulfil a predefined fraction of his contract obligations by paying a certain amount $B$.

2. In addition to this formal contract, the parties agree that the principal should make use of this clause if $V$ mets a certain target $\underline{V}$.

3. Defection is understood as

(a) the agent not delivering $\underline{V}$ or

(b) the principal not paying $B$ although $\underline{V}$ has been achieved.

By stipulating an exchange of payments between the formal and the relational contract, the mandatory payment which is due if the principal decides to defect can be held at the high level of the purely formal contract. This enhances the credibility of the relational contract. For example, if the verifiable measure is almost perfectly 
congruent, there is only little difference between the mandatory payment and the voluntary payment against which it is exchanged. Consequently, the principal bears only little incremental costs if he pays the bonus. Since only these incremental costs have to be traded off against the benefits from an ongoing relational contract, the latter is more likely to be credible. ${ }^{8}$

The following analysis studies the benefits from such an agreement. At first, I analyze the benchmark case of an additional relational contract, as considered in Baker et al. (1994). Their main findings are reproduced and formulated in the present notation, thereby providing a more concise description in terms of the congruity index derived in section 2 . I then turn to the more elaborate agreement, first considering the hypothetical case of a bonus based on $V-P^{0}$, and then proving that the same result can be achieved with an opting-out clause in the formal contract. By comparison of the results, it emerges that relational contracts are credible for a much wider range of parameters when the exchange is applied.

\subsection{A bonus offered in addition to a piece rate}

To study the effects of a relational contract, in the following I assume that the stage game analyzed in section 2 is repeated infinitely often. In this subsection, it is assumed that the principal accounts for the unverifiable information by offering a hybrid contract of the form

$$
S=s_{b}+s_{p} P+s_{v} V
$$

\footnotetext{
${ }^{8} \mathrm{~A}$ similar approach is taken in Pearce in Stacchetti (1998), who also consider the renegotiation of a formal contract in the stage game. In their model, however, the instrument is solely used for risk sharing purposes, whereas the present model focuses on congruity aspects.
} 
which adds payments from a formal agreement $s_{b}+s_{p} P$ and and a relational contract $s_{v} V$. The agent's expected utility from such a contract is $\mathrm{E} U(S, a)=s_{b}+s_{p} \mathbf{y}^{\prime} \mathbf{a}+$ $s_{v} \mathbf{d}^{\prime} \mathbf{a}-C(\mathbf{a})$. If the agent trusts in the principal's promise to pay $s_{v} V$, his effort will be

$$
\mathbf{a}\left(s_{p}, s_{v}\right)=s_{p} \mathbf{y}+s_{v} \mathbf{d}
$$

Obviously, under the assumption that $P$ is not perfectly congruent, the first-best effort allocation can be obtained by this contract only for $s_{p}=0$, a purely relational contract.

More generally, I am are interested in the optimal share parameters $s_{p}$ and $s_{v}$ of the two contracts. Given the agent's action choice (4), the principal maximizes

$$
\begin{aligned}
\mathrm{E} U^{P}\left(\mathbf{a}^{h}\right) & =V\left(\mathbf{a}^{h}\right)-C\left(\mathbf{a}^{h}\right)-U^{R} \\
& =\mathbf{d}^{\prime}\left(s_{p} \mathbf{y}+s_{v} \mathbf{d}\right)-\left(s_{p} \mathbf{y}+s_{v} \mathbf{d}\right)^{\prime}\left(s_{p} \mathbf{y}+s_{v} \mathbf{d}\right) / 2-U^{R} .
\end{aligned}
$$

The optimal value of $s_{p}$, given the bonus $s_{v}$, is

$$
s_{p}\left(s_{v}\right)=\left(1-s_{v}\right) \frac{\mathbf{d}^{\prime} \mathbf{y}}{\mathbf{d}^{\prime} \mathbf{y}}=\left(1-s_{v}\right) s_{p}^{0} .
$$

Explicit incentives are identical to those in a purely formal contract, reduced to the fraction $1-s_{v}$ not covered by the relational contract. By substitution of $s_{p}$, the agent's action $\mathbf{a}^{h}=s_{v} \mathbf{d}+\left(1-s_{v}\right) \mathbf{y}^{0}$ is a convex combination of the first-best action and the second-best action. From this, the principal's objective (5) after rearrangement becomes ${ }^{9}$

$$
\mathrm{E} U^{P}\left(\mathbf{a}^{h}\right)=\Pi^{0}+s_{v}\left(2-s_{v}\right)\left[\Pi^{F B}-\Pi^{0}\right]-U^{R}=\Pi^{F B}\left[\phi+s_{v}\left(2-s_{v}\right)(1-\phi)-\hat{\phi}\right]
$$

\footnotetext{
${ }^{9}$ For a derivation, see appendix A.
} 
The principal's utility is maximized for $s_{v}=1$. Hence, he will choose the highest share rate $s_{v} \in[0,1]$ for which the subjective reward is credible. To that purpose, the payable bonus

$$
\begin{aligned}
s_{v} V & =s_{v} \mathbf{d}^{\prime}\left(s_{v} \mathbf{d}+\left(1-s_{v}\right) \mathbf{y}^{0}\right)=s_{v}\left(\mathbf{y}^{0}\right)^{\prime} \mathbf{y}^{0}+s_{v}^{2}\left(\mathbf{d}^{\prime} \mathbf{d}-\left(\mathbf{y}^{0}\right)^{\prime} \mathbf{y}^{0}\right) \\
& =2 s_{v}\left[\Pi^{0}+s_{v}\left(\Pi^{F B}-\Pi^{0}\right)\right]=2 s_{v} \Pi^{F B}\left[\phi+s_{v}(1-\phi)\right]
\end{aligned}
$$

must not exceed the net present value of the benefits generated by the hybrid contract. As mentioned above, these benefits differ according to the principal's fallback position. This is either $\Pi^{0}-U^{R}$ (for a continued formal contract) or 0 (for ceasing production), whichever is greater. Thus, the principal in general gains

$$
\begin{aligned}
\Delta U^{P} & =\mathrm{E}^{P}\left(a^{h}\right)-\max \left\{\Pi^{0}-U^{R}, 0\right\} \\
& =s_{v}\left(2-s_{v}\right)\left[\Pi^{F B}-\Pi^{0}\right]+\min \left\{\Pi^{0}-U^{R}, 0\right\} \\
& =\Pi^{F B}\left[s_{v}\left(2-s_{v}\right)(1-\phi)+\min \{\phi-\hat{\phi}, 0\}\right]
\end{aligned}
$$

from an ongoing relational contract in each period.

In calculating the optimal bonus parameter $s_{v}$, I focus on the case of a valuable purely formal contract, for which Baker et al. (1994) derive the counterintuitive result that the existence of a sufficiently congruent verifiable performance measure may rule out any subjective reward. It is derived from the credibility constraint

$$
\sum_{t=1}^{\infty} \Delta U^{P}(1+r)^{-t}=\frac{\Delta U^{P}}{r} \geq s_{v} V
$$

that the net present value of the principal's periodical benefits have to cover the 
payable bonus. The optimal level of $s_{v}$ is ${ }^{10}$

$$
s_{v}= \begin{cases}1 & \text { for } \phi \leq 1-2 r \\ 2 \frac{1-(1+r) \phi}{(1-\phi)(1+2 r)} & \text { for } 1-2 r<\phi \leq 1 /(1+r) \\ 0 & \text { for } \phi>1 /(1+r) .\end{cases}
$$

By inspection of (9), the contract structure derived by Baker et al. (1994, p. 17) can be approved: A purely relational contract is offered for $\phi<1-2 r$. Under this contract, the first-best solution is obtained. For $1-2 r<\phi \leq 1 /(1+r)$, both a formal and a relational contract are agreed upon, with a decreasing portion of subjective rewards. And most importantly, no relational contract is credible if $\phi>1 /(1+r)$, in which case the second-best solution as under a purely formal contract is achieved.

The last, negative result of course only holds for the case of a valuable formal contract. If $\phi<\hat{\phi}$, the principal will not offer a formal contract after a potential defection, and a higher congruity cannot restrict the use of subjective rewards. It only reduces the payable bonus and is therefore always beneficial. ${ }^{11}$

Beyond the type of the applied contract, it is of interest whether congruity may also diminish the principal's utility. This seems apparent because the bonus rate in (9) is decreasing in $\phi$ for the interior solution. Actually, the conjecture can be approved by a formal analysis:

Proposition 1 If a relational contract is offered in addition to a formal contract and the purely formal contract is valuable, the principal's expected net utility $E U^{P}$ is

1. constant in $\phi$ for $\phi<1-2 r$,

\footnotetext{
${ }^{10}$ The derivation is in appendix B.

${ }^{11}$ Since these properties are obviously in line with the findings of Baker et al., I will not analyze this situation in detail here.
} 
2. decreasing in $\phi$ for $1-2 r \leq \phi<1 /(1+r)$, and

3. increasing in $\phi$ for $\phi>1 /(1+r)$.

Summarizing, we see that the main findings of Baker et al. also hold in the present framework. It is therefore well-suited to prove the effects of the contract modification outlined in subsection 3.1. A formal analysis will be done in the following subsection.

\subsection{A bonus offered in exchange to a piece rate}

The relational contract considered in the previous section makes use of only part of the available information. In general, not only $V$, but also $P$ could be applied to determine the amount to be paid as a bonus.

To see most clearly the effects of using the complete information, suppose for a moment that $P$ can be observed without noise. In that case, a relational contract can be based on $V$ and $P$ in the same manner as $V$ was used in the previous analysis. A payment $S=s_{b}+s_{p} P+s_{v} V+s_{p r} P$ is stipulated, resulting in an action $\mathbf{a}\left(s_{p}, s_{v}, s_{p r}\right)=\left(s_{p}+s_{p r}\right) \mathbf{y}+s_{v} \mathbf{d}$. According to the above analysis, the principal will choose $s_{p}$ and $s_{p r}$ such that $s_{p}+s_{p r}=\left(1-s_{v}\right) s_{p}^{0}$ : The implemented action again

will be a convex combination of $\mathbf{a}^{F B}=\mathbf{d}$ and $\mathbf{a}^{0}=\mathbf{y}^{0}$. The remaining degree of freedom in choosing $s_{p}$ and $s_{p r}$ is exploited to reduce the expected bonus $s_{v} V+s_{p r} P$ to a minimum. The solution is given in the following lemma:

Lemma 2 If $P$ is subjectively assessed without noise, the optimal hybrid contract has the following properties:

1. The formal contract is identical to the purely formal contract $s_{p}^{0}$.

2. The relational contract is based on $V-P^{0}$. 
The principal's utility from this contract, given $s_{v}$, is identical to his utility in section 3.2. The required bonus, however, may be reduced dramatically. Its expected vaule is ${ }^{12}$

$$
s_{v}\left(\mathbf{d}-\mathbf{y}^{0}\right)^{\prime} \mathbf{a}=2 s_{v}^{2}\left(\Pi^{F B}-\Pi^{0}\right)=2 s_{v}^{2} \Pi^{F B}(1-\phi)
$$

The bonus now refers only to the extra profit generated by the relational agreement. Since the action induced by the second-best pure formal contract alone is the one closest to the first-best action, a combination with this contract reduces the bonus payment to a minimum, regardless which action $s_{v} \mathbf{d}+\left(1-s_{v}\right) \mathbf{y}^{0}$ is implemented. Compared to (7), the bonus is reduced particularly in situations where the verifiable performance measure has a high congruity. Consequently, a higher share rate $s_{v}$ can be determined. Its optimal value is ${ }^{13}$

$$
s_{v}= \begin{cases}1 & \text { for } r \leq \frac{1}{2} \\ \frac{2}{1+2 r} & \text { for } r>\frac{1}{2} .\end{cases}
$$

The optimal bonus is independent of the congruity $\phi$ because the relational contract refers to only that part of the desired action which has not been covered by the formal contract. That way, the bonus is reduced by the same amount to which the principal's fallback position on the other hand is improved. Concerning the credibility of the voluntary payment, the two effects exactly balance.

The contract modification turns both findings of subsection 3.2. The first contrast can directly be seen from (11): A relational contract is now credible for any level of congruity $\phi$ and any discount rate $r$. Of course, the applied bonus rate might

\footnotetext{
${ }^{12} \mathrm{~A}$ derivation is in appendix $\mathrm{E}$.

${ }^{13} \mathrm{~A}$ derivation is in appendix $\mathrm{F}$.
} 
be rather low. But since the marginal bonus required to induce a deviation from the second-best action $\mathbf{a}^{0}$ is zero, $s_{v}$ will always be positive. The second contrast follows upon substitution of (11) in the principal's profit (6), and is given in the following lemma:

Lemma 3 Suppose $P$ is subjectively assessed without noise and a purely formal contract is valuable. If both $V$ and $P$ are used in the relational contract, the principal's expected net utility is nondecreasing in the congruity $\phi$ of the verifiable performance measures.

Thus, if $P$ were observed without noise, none of the previous negative results would remain valid under a contract which uses all available information. I use this finding as a benchmark for the more realistic scenario that $P$ is only a noisy measure of the agent's performance. In that case, a relational contract based on $v-P^{0}$ becomes critical because due to the distribution of $\epsilon$, very low levels of $P$ could result from the performance measure's noisiness. Under the proposed performance measure $V-P^{0}$, this would entail an extremely high bonus be to paid. Consequently, any promised reward would be refused with a certain probability, and the relational agreement would collapse.

As outlined in subsection 3.1, however, the same effect can be achieved by referring to the payment $s_{v} V$ instead of directly referring to $P$. If this is done before the performance measure has been realized, the principal will trade off the bonus against the expected payment from the formal contract, which, contrary the realized amount, for a given action is bounded from above.

The most apparent way to mimic the elaborate contract would be to offer a formal contract $s_{b}+s_{p}^{0} P$ at date $t .0$, and to allow the principal to compensate a fraction $s_{v}$ of the variable payment $s_{p}^{0} P$ by paying $s_{v} V$ at date $t .2$ instead. Unfortu- 
nately, this agreement is not feasible because due to the missing verifiability of $V$, the exchange cannot be substantiated in the formal contract. The formal agreement, however, is crucial because otherwise the principal could claim a very low level of $V$ in order to exchange the payment from the formal contract against a minimal bonus payment.

Therefore, the opting-out clause in the formal contract has to determine a fixed amount $B$ which can be paid in exchange to a fraction $s_{v}$ of the variable payment from the formal contract. In addition, the parties informally agree upon a target $\underline{V}$ of $V$ for which the bonus is due. If they do so, implementation of a certain action $\mathbf{a}^{h}=s_{v} \mathbf{d}+\left(1-s_{v}\right) \mathbf{y}^{0}$ requires a target $\underline{V}=s_{v} \mathbf{d}^{\prime} \mathbf{a}^{h}$ of $V$. The promised bonus must be such that the agent prefers $\mathbf{a}^{h}$ to $\mathbf{a}^{0}$, his action induced by the formal contract alone. By a comparison of the agent's utility

$\mathrm{E} U^{A}\left(\mathbf{a}^{h}\right)=s_{b}+\left(1-s_{v}\right) s_{p}^{0} \mathbf{y}^{\prime}\left(s_{v} \mathbf{d}+\left(1-s_{v}\right) \mathbf{y}^{0}\right)+B-\left(s_{v} \mathbf{d}+\left(1-s_{v}\right) \mathbf{y}^{0}\right)^{\prime}\left(s_{v} \mathbf{d}+\left(1-s_{v}\right) \mathbf{y}^{0}\right) / 2$

under the required action $\mathbf{a}^{h}$ to his expected utility

$$
\mathrm{E} U^{A}\left(\mathbf{a}^{0}\right)=s_{b}+\left(\mathbf{y}^{0}\right)^{\prime} \mathbf{y}^{0}-\left(\mathbf{y}^{0}\right)^{\prime} \mathbf{y}^{0} / 2
$$

under his optimal action $\mathbf{a}^{0}=\mathbf{y}^{0}$ if he waives the bonus, the minimal bonus

$$
\underline{B} \equiv s_{v}\left(\mathbf{y}^{0}\right)^{\prime} \mathbf{y}^{0}+\frac{s_{v}}{2}\left[\mathbf{d}^{\prime} \mathbf{d}-\left(\mathbf{y}^{0}\right)^{\prime} \mathbf{y}^{0}\right]
$$

required to implement $\mathbf{a}^{h}$ can be determined. Although this term slightly differs from the amount (7) required without the opting-out clause, ${ }^{14}$ it seems to have the same undesirable properties with respect to credibility: Since the marginal bonus

\footnotetext{
${ }^{14} \mathrm{I}$ comment on that difference in detail below.
} 
$\mathrm{d} \underline{B} / \mathrm{d} s_{v}$ required for a divergence from the second-best action $\mathbf{a}^{0}$ is positive, there always exist discount rates $r<\infty$ for which no relational contract is credible, regardless the congruity $\phi$ of the verifiable performance measure. Thus, at first glance, there is no obvious improvement. However, the important point here is that not the absolute bonus is crucial, but the incremental cost the principal incurs by paying the bonus. In this respect, under the opting-out clause not only the bonus $B$, but also the expected amount $s_{v}\left(\mathbf{y}^{0}\right)^{\prime} \mathbf{y}^{0}$ saved from the formal obligations has to be taken into account. In consideration of these savings, the principal's extra cost from the bonus is

$$
\underline{B}-s_{v}\left(\mathbf{y}^{0}\right)^{\prime} \mathbf{y}^{0}=\frac{s_{v}}{2}\left[\mathbf{d}^{\prime} \mathbf{d}-\left(\mathbf{y}^{0}\right)^{\prime} \mathbf{y}^{0}\right]
$$

which is half the amount which was due under a relational contract based on $V-P^{0}$. Consequently, the relational contract is credible to an even greater extent. The bonus rate is ${ }^{15}$

$$
s_{v}= \begin{cases}1 & \text { for } r \leq 1 \\ \frac{2}{1+r} & \text { for } r>1\end{cases}
$$

from which the main result of this paper can be derived:

Proposition 2 If a relational contract is offered in exchange to part of a formal contract and the purely formal contract is valuable,

1. a hybrid contract is credible for any discount rate $r$ and any level $\phi$ of congruity, and

2. the principal's expected net utility is increasing in $\phi$ for any level of congruity.

Thus, by application of the opting-out clause the principal does even better than under the contract based on $V-P^{0}$. This fact is due to the different contract type

\footnotetext{
${ }^{15}$ The derivation is in appendix $\mathrm{H}$.
} 
required by the opting-out clause: while the contract based on $V-P^{0}$ is a linear function of the aggregate performance measure, a bonus-type contract is used under the opting-out clause. By this means, the payment is reduced to one half because only the cost of the additional effort $s_{v}\left(\mathbf{d}-\mathbf{y}^{0}\right)$ has to be compensated. A linear contract, in contrast, generally pays two times the cost, provided the cost function is quadratic. Therefore, the same result as in proposition 2 could have been achieved by a bonus-type relational contract based on $V-P^{0}$.

Halving the bonus (9) in the initial contract based on $V$, in contrast, would not essentially change the results of subsection 3.2. Since this bonus covers not only the cost of the additional effort $s_{v}\left(\mathbf{d}-\mathbf{y}^{0}\right)$, but also has to actuate the effort $\left(1-s_{v}\right) \mathbf{y}^{0}$ not driven by the (reduced) formal contract, the marginal bonus required for a divergence from the action induced by the formal contract is still positive. Thus, although the bonus would be credible for a wider range of discount rates and congruity levels, there would still exist parameters $r$ and $\phi$ for which no relational contract is credible. Only by restricting the payment to the cost incurred by a divergence from the optimal pure formal contract, the general credibility is obtained.

To complete the analysis of the hybrid contract with an opting-out clause, I finally study the case $\phi<\hat{\phi}$ for which a purely formal contract is not valuable for the principal. In this case, his benefit (8) from an ongoing contract is $\Pi^{F B}\left[s_{v}\left(2-s_{v}\right)(1-\phi)-(\hat{\phi}-\phi)\right]$, which is smaller than the amount under a beneficial pure formal contract. Nevertheless, it is increasing in the congruity $\phi$. Since on the other hand the incremental cost (12) decreases in $\phi$, higher levels of $s_{v}$ can 
be stipulated for higher levels of congruity. The optimal share rate is ${ }^{16}$

$$
s_{v}= \begin{cases}\frac{1}{1+r}+\frac{\sqrt{(1-\phi)(1+r \phi-\hat{\phi}(1+r))}}{(1-\phi)(1+r)} & \text { for } \phi \geq \frac{(1+r) \hat{\phi}-1}{r} \\ 0 & \text { for } \phi<\frac{(1+r) \hat{\phi}-1}{r} .\end{cases}
$$

If the congruity exceeds a critical level, a hybrid contract becomes valuable. This contract includes a minimum level $\underline{s}_{v}=1 /(1+r)$ of subjective rewards, and a formal contract, which would not be beneficial on its own. For higher levels of congruity, the share rate increases and for $\phi \rightarrow \hat{\phi}$ achieves the optimal level under a valuable formal contract. Thus, in this respect, the results are conform with those of Baker et al. Summarizing the effects with the findings of proposition 2, the advantage of a more congruent verifiable performance measure can generally be stated:

Corrollary 1 If the principal applies the opting-out clause in the hybrid contract, he always (weakly) benefits from a higher congruity of the verifiable performance measures.

Regardless of his fallback position, the principal will always benefit from a less distorted performance measure. Thus, by considering the more elaborate contract, the paradox of the existing literature could be cleared.

\section{Conclusion}

In this paper, it was shown that under a proper combination, formal and relational contract are complementary for incentive contracting. The key feature of the relational contract is that it not only refers to the unverifiable information, but also makes use of the performance measures applied in formal contracting. That way,

\footnotetext{
${ }^{16} \mathrm{~A}$ derivation is in appendix $\mathrm{J}$.
} 
implicit incentives can be reduced to that part of the desired action which cannot be induced by the formal contract. As a consequence, the payable bonus is reduced, which makes the relational contract credible for a much wider range of discount factors.

The use of all available information for the relational contract was made possible by the contractual arrangement of an opting-out clause. Killing this option, the principal reduces his obligations from the formal contract, which in turn reduces his relevant cost from the bonus payment. Crucial to the use of this instrument is the assumption that the principal is not aware of the agent's financial performance when he decides to pay the bonus. Consequently, the timeliness of the nonfinancial information substantiating the relational contract becomes extremely important. Only if this information is available at a point of time which is foreseen when the contract is written, the principal's ignorance of his hard obligations can be exploited to improve the credibility of the relational agreement.

As a practical implication, norms for the preparation of strategic performance information may become an important aspect for the provision of incentives. On the one hand, such norms may improve the verifiability of nonfinancial performance measures, resulting in a more congruent aggregate of verifiable measures. As the analysis has shown, the agency will always benefit from this improvement. On the other hand, norms may be essential to the use of the remaining unverifiable measures if they fix periods for which the data has to be collected. 


\section{A Derivation of equation (6)}

With $\mathbf{a}^{h}=s_{v} \mathbf{d}+\left(1-s_{v}\right) \mathbf{y}^{0}$, the principal's expected utility (5) becomes

$$
\begin{aligned}
\mathrm{E} U^{P}\left(\mathbf{a}^{h}\right) & =\mathbf{d}^{\prime}\left(s_{v} \mathbf{d}+\left(1-s_{v}\right) \mathbf{y}^{0}\right)-\frac{1}{2}\left[\left(s_{v} \mathbf{d}+\left(1-s_{v}\right) \mathbf{y}^{0}\right)^{\prime}\left(s_{v} \mathbf{d}+\left(1-s_{v}\right) \mathbf{y}^{0}\right)\right]-U^{R} \\
& =s_{v} \mathbf{d}^{\prime} \mathbf{d}+\left(1-s_{v}\right) \mathbf{d}^{\prime} \mathbf{y}^{0}-\frac{1}{2}\left[\left(s_{v}^{2} \mathbf{d}^{\prime} \mathbf{d}+2 s_{v}\left(1-s_{v}\right) \mathbf{d} \mathbf{y}^{0}+\left(1-s_{v}\right)^{2}\left(\mathbf{y}^{0}\right)^{\prime} \mathbf{y}^{0}\right]-U^{R}\right.
\end{aligned}
$$

Taking into account that $\mathbf{d}^{\prime} \mathbf{y}^{0}=\left(\mathbf{y}^{0}\right)^{\prime} \mathbf{y}^{0}$, this is equal to

$$
\begin{aligned}
\mathrm{E} U^{P}\left(\mathbf{a}^{h}\right) & =s_{v} \mathbf{d}^{\prime} \mathbf{d}+\left(1-s_{v}\right)\left(\mathbf{y}^{0}\right)^{\prime} \mathbf{y}^{0}-\frac{1}{2}\left[\left(s_{v}^{2} \mathbf{d}^{\prime} \mathbf{d}+\left(1-s_{v}^{2}\right)\left(\mathbf{y}^{0}\right)^{\prime} \mathbf{y}^{0}\right]-U^{R}\right. \\
& =\frac{s_{v}\left(2-s_{v}\right)}{2} \mathbf{d}^{\prime} \mathbf{d}+\frac{\left(1-s_{v}\right)^{2}}{2}\left(\mathbf{y}^{0}\right)^{\prime} \mathbf{y}^{0}-U^{R} \\
& =\frac{\left(\mathbf{y}^{0}\right)^{\prime} \mathbf{y}^{0}}{2}+s_{v}\left(2-s_{v}\right)\left[\frac{\mathbf{d}^{\prime} \mathbf{d}}{2}-\frac{\left(\mathbf{y}^{0}\right)^{\prime} \mathbf{y}^{0}}{2}\right]-U^{R} \\
& =\Pi^{0}+s_{v}\left(2-s_{v}\right)\left[\Pi^{F B}-\Pi^{0}\right]-U^{R}
\end{aligned}
$$

\section{B Derivation of equation (9)}

Credibility of the bonus payment requires $r s_{v} V \leq \Delta U^{P}$, i.e.,

$$
2 r s_{v} \Pi^{F B}\left[\phi+s_{v}(1-\phi)\right] \leq \Pi^{F B}\left[s_{v}\left(2-s_{v}\right)(1-\phi)+\min \{\phi-\hat{\phi}, 0\}\right] .
$$

The solution to this quadratic inequality is

$$
0 \leq s_{v} \leq 2 \frac{(1-\phi(1+r))}{(1-\phi)(1-2 r)}
$$

No solution exists if the right term is negative, which is the case for $\phi>1 /(1+r)$.

In this case, no subjective reward is promised, and $s_{v}=0$. If $\phi \leq 1-2 r$, the right term is larger than one, and the credibility constraint is not binding. In this case, 
the first-best share rate $s_{v}=1$ is chosen. In all other cases, $s_{v}$ equals the upper bound in (15).

\section{Proof of proposition 1}

Upon substitution of (9), the principal's profit (6) becomes

$$
\mathrm{E} U^{P}= \begin{cases}\Pi^{F B}-U^{R} & \text { for } \phi \leq 1-2 r \\ \Pi^{F B}\left[\phi+4 r \frac{(2-\phi)(1-\phi(1+r))}{(1+2 r)^{2}(1-\phi)}\right]-U^{R} & \text { for } 1-2 r<\phi \leq 1 /(1+r) \\ \Pi\left(\mathbf{s}^{0}\right)=\phi \Pi^{F B}-U^{R} & \text { for } \phi>1 /(1+r) .\end{cases}
$$

Differentiating with respect to $\phi$ yields

$$
\frac{\partial}{\partial \phi} \mathrm{E} U^{P}= \begin{cases}0 & \text { for } \phi \leq 1-2 r \\ \Pi^{F B} \frac{(1-\phi)^{2}-4 r^{2}}{(1+2 r)^{2}(1-\phi)^{2}} & \text { for } 1-2 r<\phi \leq 1 /(1+r) \\ \Pi^{F B} & \text { for } \phi>1 /(1+r) .\end{cases}
$$

The sign of the first and the third term is obvious. The second term is negative for $\phi \geq 1-2 r$, which is always true in the relevant range of $\phi$.

\section{Proof of lemma 2}

The principal aims at minimizing the expected bonus $s_{v} \mathbf{d}^{\prime} \mathbf{a}+s_{p r} \mathbf{y}^{\prime} \mathbf{a}$, which for $\mathbf{a}=\left(s_{p}+s_{p r}\right) \mathbf{y}+s_{v} \mathbf{d}$ becomes

$$
\left.s_{v} \mathbf{d}^{\prime}\left(s_{p}+s_{p r}\right) \mathbf{y}+s_{v} \mathbf{d}\right)+s_{p r} \mathbf{y}^{\prime}\left(\left(s_{p}+s_{p r}\right) \mathbf{y}+s_{v} \mathbf{d}\right) .
$$


Differentiation with respect to $s_{p}$ yields the first-order condition

$$
s_{v} \mathbf{d}^{\prime} \mathbf{y}+s_{p r} \mathbf{y}^{\prime} \mathbf{y}=0
$$

The respective optimal level $s_{p r}=-s_{v} \mathbf{d}^{\prime} \mathbf{d} / \mathbf{y}^{\prime} \mathbf{y}=-s_{v} s_{p}^{0}$ is applied to proof part 1 of the lemma by $s_{v} V+s_{p r} P=s_{v} V-s_{v} s_{p}^{0} P=s_{v}\left(V-P^{0}\right)$.

Part 2 of the proposition is derived by substitution of $s_{p r}$ in the above condition $s_{p}+s_{p r}=1-s_{v}$ from which $s_{p}$ is $s_{p}=\left(1-s_{v}\right) s_{p}^{0}-s_{p r}=s_{p}^{0}$.

\section{E Derivation of equality (10)}

$$
\begin{aligned}
s_{v}\left(\mathbf{d}-\mathbf{y}^{0}\right)^{\prime} \mathbf{a} & =s_{v}\left(\mathbf{d}-\mathbf{y}^{0}\right)\left(s_{v} \mathbf{d}+\left(1-s_{v}\right) \mathbf{y}^{0}\right) \\
& =s_{v}\left(\mathbf{d}-\mathbf{y}^{0}\right)\left(\mathbf{y}^{0} s_{v}\left(\mathbf{d}-\mathbf{y}^{0}\right)\right. \\
& =s_{v}^{2}\left(\mathbf{d}-\mathbf{y}^{0}\right)^{\prime}\left(\mathbf{d}-\mathbf{y}^{0}\right) \\
& =s_{v}^{2}\left[\mathbf{d}^{\prime} \mathbf{d}-\left(\mathbf{y}^{0}\right)^{\prime} \mathbf{y}^{0}\right] \\
& =2 s_{v}^{2}\left(\Pi^{F B}-\Pi^{0}\right) .
\end{aligned}
$$

\section{F Derivation of equation 11}

Credibility of the bonus payment requires $r s_{v} V \leq \Delta U^{P}$, i.e.,

$$
2 r s_{v}^{2} \Pi^{F B}(1-\phi) \leq \Pi^{F B}\left[s_{v}\left(2-s_{v}\right)(1-\phi)+\min \{\phi-\hat{\phi}, 0\}\right] .
$$

The solution to this quadratic inequality is

$$
0 \leq s_{v} \leq \frac{2}{1+2 r}
$$


If $r l e 1 / 2$, the right term is larger than one, and the credibility constraint is not binding. In this case, the first-best share rate $s_{v}=1$ is chosen. In all other cases, $s_{v}$ equals the upper bound in (16).

\section{G Proof of lemma 3}

Upon substitution of (11), the principal's profit (6) becomes

$$
\mathrm{E} U^{P}= \begin{cases}\Pi^{F B}-U^{R} & \text { for } r \leq \frac{1}{2} \\ \Pi^{F B}\left[\phi+\frac{8 r}{(1+2 r)^{2}}(1-\phi)\right] & \text { for } r>\frac{1}{2}\end{cases}
$$

Differentiating with respect to $\phi$ yields

$$
\frac{\partial}{\partial \phi} \mathrm{E} U^{P}= \begin{cases}0 & \text { for } r \leq \frac{1}{2} \\ \Pi^{F B}\left[1-\frac{8 r}{1+2 r}\right] & \text { for } r>\frac{1}{2}\end{cases}
$$

The second term is positive if $(1-2 r)^{2}>0$, which is always true in the relevant range of $r$.

\section{H Derivation of equation 13}

Credibility of the bonus payment requires $r s_{v} V \leq \Delta U^{P}$, i.e.,

$$
r s_{v}^{2} \Pi^{F B}(1-\phi) \leq \Pi^{F B}\left[s_{v}\left(2-s_{v}\right)(1-\phi)\right] .
$$


The solution to this quadratic inequality is

$$
0 \leq s_{v} \leq \frac{2}{1+r}
$$

If $r \leq 1$, the right term is larger than one, and the credibility constraint is not binding. In this case, the first-best share rate $s_{v}=1$ is chosen. In all other cases, $s_{v}$ equals the upper bound in (18).

\section{Proof of proposition 2}

1. Obvious from (13).

2. Upon substitution of $s_{v}$, the principal's expected utility (6) becomes

$$
\mathrm{E} U^{P}= \begin{cases}\Pi^{F B}-U^{R} & \text { for } r \leq \frac{1}{2} \\ \Pi^{F B}\left[\phi+\frac{1+2 r}{(1+r)^{2}}(1-\phi)\right] & \text { for } r>\frac{1}{2}\end{cases}
$$

Differentiating with respect to $\phi$ yields

$$
\frac{\partial}{\partial \phi} \mathrm{E} U^{P}= \begin{cases}0 & \text { for } r \leq \frac{1}{2} \\ \Pi^{F B}\left[1-\frac{1+2 r}{1+r}^{2}\right] & \text { for } r>\frac{1}{2}\end{cases}
$$

The second term is nonnegative for $r^{2} \geq 0$, which is always the case. 


\section{J Derivation of equation 14}

The credibility constraint is

$$
r s_{v}^{2} \Pi^{F B}(1-\phi) \leq \Pi^{F B}\left[s_{v}\left(2-s_{v}\right)(1-\phi)-(\hat{\phi}-\phi)\right]
$$

This is fulfilled for

$$
\frac{1}{1+r}-\frac{\sqrt{(1-\phi)(1+r \phi-\hat{\phi}(1+r))}}{(1-\phi)(1+r)} \leq s_{v} \leq \frac{1}{1+r}+\frac{\sqrt{(1-\phi)(1+r \phi-\hat{\phi}(1+r))}}{(1-\phi)(1+r)}
$$

Such $s_{v}$ exist if the radicand in (20) is nonnegative, which holds for $r \leq(1-\hat{\phi}) /(\hat{\phi}-\phi)$ or $\phi \geq[(1+r) \hat{\phi}-1] / r$.

\section{K Proof of Corollary 1}

For $\phi \geq \hat{\phi}$, the claim directly follows from proposition 2. For $\phi<\hat{\phi}$, an explicit computation of $\mathrm{EU}^{P}$ is complicated. Instead, consider the principal's problem of choosing the parameter $s_{v}$ in order to maximize his expected profit (6) subject to the credibility constraint, which takes the form:

$$
\begin{aligned}
\max _{s_{v}} \mathrm{E} U^{P}= & \Pi^{F B}\left[s_{v}\left(2-s_{v}\right)(1-\phi)-(\hat{\phi}-\phi)\right] . \\
\text { s.t. } & r s_{v}^{2} \Pi^{F B}(1-\phi) \leq \Pi^{F B}\left[s_{v}\left(2-s_{v}\right)(1-\phi)-(\hat{\phi}-\phi)\right] .
\end{aligned}
$$


By the envelope theorem, the impact of congruity is given by the partial derivative of the Lagrangian with respect to $\phi$,

$$
\frac{\mathrm{dE} U^{P}}{\mathrm{~d} \phi}=\frac{\partial \mathcal{L}}{\partial \phi}=\left(1-s_{v}\right)^{2} \Pi^{F B}(1+\lambda)+\lambda r \Pi^{F B} s_{v}^{2},
$$

which is nonnegative because $\lambda \geq 0$.

\section{References}

[1] Abreu, D. "On the theory of infinitely repeated games with discounting." Econometrica 56, 2 (1988): 383-396.

[2] BAKer, G. P. "The Use of Performance Measures in Incentive Contracting." American Economic Review 90, 2 (2000): 415-420.

[3] BAKer, G. P. "Distortion and Risk in Optimal Incentive Contracts." The Journal of Human Resources 37, 4 (2002): 728-751.

[4] Baker, G., R. Gibbons and K. J. Murphy. "Subjective Performance Measures in Optimal Incentive Contracts." Quarterly Journal of Economics 109 (1994): 1125-1156.

[5] Baker, G., R. Gibbons and K. J. Murphy. "Relational Contracts and the Theory of the Firm." Quarterly Journal of Economics 117 (2002): 39-83.

[6] Banker, R.D And S. Datar. "Sensitivity, Precision, and Linear Aggregation of Signals for Performance Evaluation." Journal of Accounting Research 27, 1 (1989): $21-39$. 
[7] Budde, J.. "Performance measure congruity and the balanced scorecard." Working paper, University of Bonn.

[8] Datar, S. M., S. C. Kulp and R. A. Lambert. "Balancing Performance Measures." Journal of Accounting Research 39, 1 (2001): 75-92.

[9] Feltham, G. A. And J. Xie. "Performance Measure Congruity and Diversity in Multi-Task Principal/Agent Relations." The Accounting Review 69, 3 (1994): 429-453.

[10] Feltham, G.A. And J. Wu "Public Reports, Information Acquisition by Investors, and Management Incentives." Review of Accounting Studies 5 (2000): 155-190.

[11] Holmström, B. And P. Milgrom. "Multitask Principal-Agent Analyses: Incentive Contracts, Asset Ownership, and Job Design." Journal of Law, Economics and Organization 7 (1991): 24-52.

[12] Kaplan, R. S. and D. P. Norton. "The Balanced Scorecard - Measures that Drive Performance". Harvard Business Review (1992): 71-79.

[13] Kaplan, R.S. and D.P. Norton. "Using the Balanced Scorecard as a Strategic Management System". Harvard Business Review (1996): 75-87.

[14] Kaplan, R.S. And D.P. NorTon. The strategy-focussed organization. Harvard Business School Publishing, 2001.

[15] Pearce, D.G. and E. Stacchetti. "The Interaction of Implicit and Explicit Contracts in Repeated Agency." Games and Economic Behavior 23 (1998): 7596. 


\begin{tabular}{cccc}
$t .0$ & $t .1$ & $t .2$ & $t .3$ \\
\hline $\begin{array}{c}\text { contract is } \\
\text { signed }\end{array}$ & $\begin{array}{c}\text { agent chooses } \\
\text { action } \mathbf{a}\end{array}$ & $\begin{array}{l}V \text { is observed } \\
\text { bonus is paid }\end{array}$ & $\begin{array}{c}P \text { is observed } \\
\text { compensation is paid }\end{array}$
\end{tabular}

Figure 1: Timeline of events 